\title{
Advances in the application of magnetic and non-magnetic superspace-group symmetry
}

\author{
B. J. Campbell, H. T. Stokes \\ Brigham Young University, Dept. of Physics \& Astronomy, Provo, Utah, USA 84602
}

branton@byu.edu

Superspace-group symmetry is essential to the unambiguous description of modulated structures, and a correct understanding of their physical properties. An exhaustive enumeration of superspace groups in up to $3+3$ dimensions were announced previously [1-2]. We now announce an exhaustive enumeration of magnetic superspace groups in up to $3+3$ dimensions (over 250,000 groups). With these tables in hand, we have developed an algorithm and tool that detects the unique superspace-group (magnetic or non-magnetic) of an arbitrary modulated structure, given the amplitudes and phases of its modulations, and identifies it in the exhaustive symmetry-group table. This capability has been integrated into both the FINSYM and ISOCIF packages of the ISOTROPY software suite [3], and to JANA2000. The ISODISTORT package [4], which uses group-representations to generate incommensurate structure models based on a given parent structure [5], now automatically identifies the unique magnetic superspace-group of each magnetically modulated child structure. Anyone can access these data sources and tools online to generate, symmetrize, transform, or otherwise explore magnetic or non-magnetic modulated structure models.

[1] H. T. Stokes, B. J. Campbell \& S. van Smaalen, Acta Cryst. A 67, 45-55 (2011).

[2] S. van Smaalen, B. J. Campbell \& H. T. Stokes, Acta Cryst. A 69, 75-90 (2013).

[3] H. T. Stokes, D. M. Hatch \& B. J. Campbell, ISOTROPY Software Suite, iso.byu.edu.

[4] B. J. Campbell, H. T. Stokes, D. E. Tanner \& D. M. Hatch, J. Appl. Cryst. 39, 607-614 (2006).

[5] H. T. Stokes, S. van Orden, \& B. J. Campbell, J. Appl Cryst. 49, 1849-1853 (2016).

Keywords: magnetic superspace group; superspace symmetry detection; incommensurate; modulated; isotropy subgroup 